\title{
Traduire
}

Une eutre perspective sur $r$ tatadciction

Revue française de la traduction

$242 \mid 2020$

Passons au vert

\section{L'information extra-financière, nouvel enjeu de traduction}

Chloé Delhom et Audrey Prost

\section{(2) OpenEdition}

1 Journals

Édition électronique

URL : http://journals.openedition.org/traduire/2012

DOI : 10.4000/traduire.2012

ISSN : 2272-9992

Éditeur

Société française des traducteurs

Édition imprimée

Date de publication : 30 juin 2020

Pagination : 68-83

ISSN : 0395-773X

Référence électronique

Chloé Delhom et Audrey Prost, «L'information extra-financière, nouvel enjeu de traduction », Traduire

[En ligne], 242 | 2020, mis en ligne le 15 juillet 2020, consulté le 20 décembre 2020. URL : http:// journals.openedition.org/traduire/2012 ; DOI : https://doi.org/10.4000/traduire.2012 


\section{L'information extra-financière, nouvel enjeu de traduction}

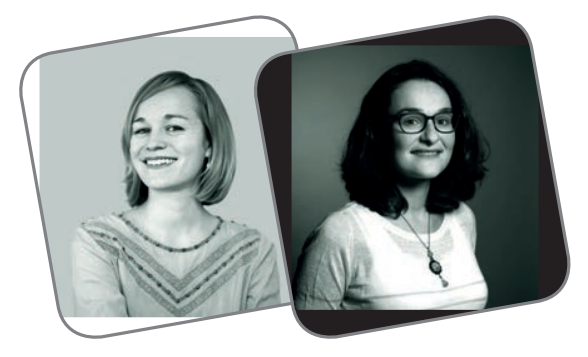

\section{Chloé Delhom, Audrey Prost}

D'expérience, nous constatons que plusieurs chemins peuvent mener vers la traduction de documents concernant le développement durable et la responsabilité sociétale des entreprises (RSE), que l'on appelle communément "information extra-financière» dans le domaine de la communication d'entreprise, et dont le contenu est de plus en plus réglementé. Nos cas personnels en attestent: une spécialisation en finance peut amener à travailler sur des parutions extra-financières; à l'inverse, un parcours en RSE est aussi l'occasion de se familiariser avec des publications plus réglementées.

Or, la multiplicité et la superposition croissante des cadres normatifs complexifient le paysage de la communication financière comme extra-financière. Comment l'information extra-financière a-t-elle progressivement trouvé sa place dans les publications périodiques des entreprises? Quelles en sont les conséquences pour les linguistes amenés à traduire ce type d'information? 
Pour comprendre ce croisement entre les sphères du financier et de la RSE, nous proposons de dresser un panorama du cadre de l'information réglementée (financière et extrafinancière) et du régime linguistique applicable aux publications institutionnelles des entreprises françaises puis de mettre en lumière quelques stratégies de traduction du vocabulaire de la RSE.

\section{L'information extra-financière dans les publications périodiques des entreprises}

\section{Qu'est-ce que l'information réglementée?}

L'information réglementée, définie par l'Autorité des marchés financiers (AMF) dans son règlement général' (art. 221-1), recouvre les informations de nature financière et relatives à la gouvernance des sociétés cotées. Ces dernières doivent être publiées de manière périodique dans un souci de transparence et d'information des publics concernés par la situation (financière) de l'entreprise, aux premiers rangs desquels les actionnaires. Elles constituent également une obligation légale pour les sociétés de droit français et comprennent:

- le rapport financier annuel (art. 222-3 RG AMF);

- le rapport financier semestriel (art. 222-4);

- les informations et rapports sur le gouvernement d'entreprise (art. 222-9 RG AMF et art. L. 22537, L. 225-68 et L. 225-235 du Code de commerce).

L'AMF rappelle que "[t]outes les sociétés cotées ayant l'AMF comme autorité compétente [...] ont désormais le choix de publier cette information soit en français, soit dans une langue usuelle en matière financière» (voir art. 221-2 RG AMF).

Elle constate néanmoins que:

[e]n pratique, [...] beaucoup de sociétés optent pour le bilinguisme et publient leur information réglementée en français et en anglais. L'AMF rappelle qu'une société de droit français qui décide d'adopter l'anglais comme langue pour ses informations périodiques devra néanmoins toujours produire annuellement 
des comptes en français au regard de ses obligations légales de dépôt au greffe [...].

L'option du bilinguisme retenue par la plupart des sociétés cotées en France participe à la fois de la bonne information des investisseurs et des contraintes liées à l'internationalisation croissante des marchés financiers. Elle est aussi source d'un flux de travail régulier et récurrent pour les traducteurs et traductrices spécialisés en finance.

\section{Le document d'enregistrement universel (ex-document de référence), incontournable publication réglementée?}

En complément de ces rapports obligatoires, la plupart des émetteurs français publiaient jusqu'à l'an dernier un document de référence (ce fameux DDR, ou registration document, dont tous les traducteurs financiers ont sûrement déjà entendu parler). Son usage était tellement répandu que l'on en oubliait presque que cette publication revêtait, contrairement au rapport financier annuel, un caractère facultatif (art. 212-13 RG AMF). D'ailleurs, l'AMF acceptait, sous certaines conditions, que ce DDR prenne la forme du rapport financier annuel destiné aux actionnaires.

Le document de référence avait émergé en France il y a une quinzaine d'années. Initialement "prospectus de marché aride et fourre-tout ${ }^{2}$ », il était devenu au fil du temps un document structuré et accessible à un plus grand public. Son contenu ne se limitait plus à la simple présentation des comptes et de la structure de gouvernance de l'entreprise: il intégrait la présentation de son activité, de sa stratégie et aussi, ces dernières années, de sa responsabilité sociétale.

Comme son nom ne l'indiquait pas en français (!), le document de référence rédigé en français pouvait être déposé (ou enregistré) auprès de l'AMF. L'émetteur pouvait alors également le déposer ou le faire enregistrer "dans une langue usuelle en matière financière [c'est-à-dire l'anglais]».

En pratique, le document de référence était devenu une publication quasiment incontournable dans l'information réglementée, car il s'agissait d'un «document de synthèse

2. wWw.labrador-company.fr/billet-du-blog-document-de-reference-urd, consulté le 29/05/2020. 
contenant l'ensemble des informations juridiques, économiques, financières et comptables d'un émetteur pour un exercice donné». Les entreprises le publiaient généralement en lieu et place du rapport financier annuel dans les quatre mois suivant la clôture de l'exercice, c'est-à-dire, pour une majorité d'entre elles, entre janvier et avril (la fameuse "saison des rapports annuels», un pic d'activité pour les traducteurs et traductrices travaillant sur ce type de publications).

Depuis juillet 2019, le document de référence a laissé place au document d'enregistrement universel (URD pour universal registration document), dont l'objectif affiché est d'amener les émetteurs européens à publier chaque année une synthèse de leur activité, de leur structure et de leur situation financière.

Or, comme le souligne l'avocate Rosetta Ferrère ${ }^{3}$,

[p]our les émetteurs français, le caractère novateur de l'URD a pu sembler, à juste titre, moins évident que pour les autres émetteurs de l'Union européenne. Et pour cause, l'URD est largement inspiré du "document de référence » bien connu et pratiqué en France depuis déjà de nombreuses années.

Si les entreprises françaises n'ont donc pas attendu la réglementation européenne pour adopter cette pratique de présentation d'informations (y compris autres que financières), I'URD permet de systématiser cette démarche de convergence entre le financier et l'extra-financier et d'en assurer l'application uniforme à l'échelle du marché européen, "au service d'une meilleure compréhension de la création de valeur et de son partage par les entreprises ${ }^{4} \gg$.

\section{Quid des informations extra-financières?}

L'idée que la création de richesse par l'entreprise doit également profiter à l'ensemble de la société semble donc gagner du terrain. À l'instar d'un nombre croissant de dirigeants d'entreprises, Franck Riboud, PDG du groupe Danone,

3. WwW.lexplicite.fr/reglement-prospectus-document-de-reference-urd-il-ny-a-quun-pas, consulté le 29/05/2020.

4. rse-reporting.com/blog-document-enregistrement-universel-de-la-convergence-dufinancier-extra-financier, consulté le 29/05/2020. 
soulignait déjà en 2009 la responsabilité de l'entreprise visà-vis de la société tout entière:

Aucun organisme ne se développe dans un milieu appauvri ou dans un désert. [...] [l]l est donc de l'intérêt même d'une entreprise de prendre soin de son environnement économique et social, ce qu'on pourrait appeler, par analogie, son "écosystème».

Ainsi, le devoir d'information des entreprises s'est progressivement étendu aux données extra-financières: environnement, responsabilité du fait des produits, emploi et relations sociales, respect des droits de l'Homme et lutte contre la corruption.

Les informations relatives à ces thématiques sont publiées dans le cadre du reporting extra-financier, autrement appelé rapport de développement durable, ou rapport RSE. Quelle que soit sa dénomination, cette nouvelle typologie de documents génère de nouveaux besoins de traduction. Elle est même parfois une source de spécialisation pour certains et certaines d'entre nous.

L'obligation de publication de données plus ou moins détaillées sur la performance RSE peut venir de la loi sur les sociétés, mais aussi du marché financier, ou encore des règles imposées aux entreprises publiques. Les entreprises visées sont majoritairement les grandes entreprises et celles cotées en Bourse. A priori, aucune loi n'inclut de sanction spécifique en cas de non-respect du reporting RSE, ce qui affaiblit quelque peu son caractère contraignant.

Le cadre normatif de la RSE se déploie à différents niveaux:

\section{- des lignes directrices internationales:}

- les lignes directrices de la Conférence des Nations unies sur le commerce et le développement (CNUCED) sur des indicateurs de la responsabilité d'entreprise dans les rapports annuels ${ }^{5}(2008)$;

- Ia Global Reporting Initiative (GRI)6 (2017);

5. unctad.org/fr/docs/iteteb20076_fr.pdf, consulté le 29/05/2020.

6. www.globalreporting.org/Pages/default.aspx, consulté le 29/05/2020. 


\section{- une directive européenne:}

- la Directive comptable européenne sur la publication des informations extra-financières (directive $n^{\circ} 2014 / 95$ );

\section{- des lois nationales:}

- dès 2001, la France était la première à rendre le reporting RSE obligatoire, avec la loi sur les nouvelles régulations économiques (NRE) pour les entreprises cotées, avant de l'élargir aux grandes entreprises avec la loi Grenelle II (2010) et l'article L. 225-102 du Code de commerce. Depuis lors, les entreprises dépassant un certain sevil de chiffre d'affaires et d'effectif doivent présenter un rapport de performance extra-financière;

- depuis le 1er août 2017, en vertu de l'ordonnance n²017-1180 qui aligne le cadre législatif français avec la directive européenne de 2014 susmentionnée, la déclaration de performance extra-financière (DPEF) remplace le rapport RSE et doit être insérée dans le rapport de gestion;

- plus récemment, la loi Pacte consacre la reconnaissance des critères extra-financiers en les intégrant au Code civil (art. 1833), qui dispose désormais que "la société est gérée dans son intérêt social en prenant en considération les enjeux sociaux et environnementaux de son activité».

\section{Et le rapport intégré, dans tout ça?}

Cette imbrication toujours plus étroite du financier et de l'extra-financier a conduit à l'émergence d'une nouvelle pratique sous l'impulsion du Comité international de l'information intégrée (IIRC): le reporting intégré, qui permet aux entreprises de produire des évaluations globales sur leurs performances financières, sociales, environnementales et de gouvernance.

Outre son caractère non contraignant, le rapport intégré se démarque des publications précédemment évoquées par sa 
concision, son approche multidimensionnelle et sa prise en compte du court, moyen et long terme.

Quant au public visé, il fait débat. D'un côté, l'Institut français des administrateurs considère que:

le public privilégié est l'investisseur. [...] Ce document n'a pas vocation à reprendre toutes les informations contenues dans le rapport de responsabilité sociale, ni à lui consacrer la majeure partie de son contenu. Ne doivent y figurer que les seules initiatives RSE qui contribuent directement et de manière claire à la création de valeur à long terme pour l'actionnaire.

D'autres mettront au contraire l'accent sur le fait que «ce rapport est utile à l'ensemble des parties prenantes intéressées», comme en témoigne le graphique suivant:

\section{Le rapport intégré : contexte et enjeux}

\section{Le rapport intégré s'adresse à toutes les parties prenantes et présente la stratégie et les performances financières et extra-financières.}

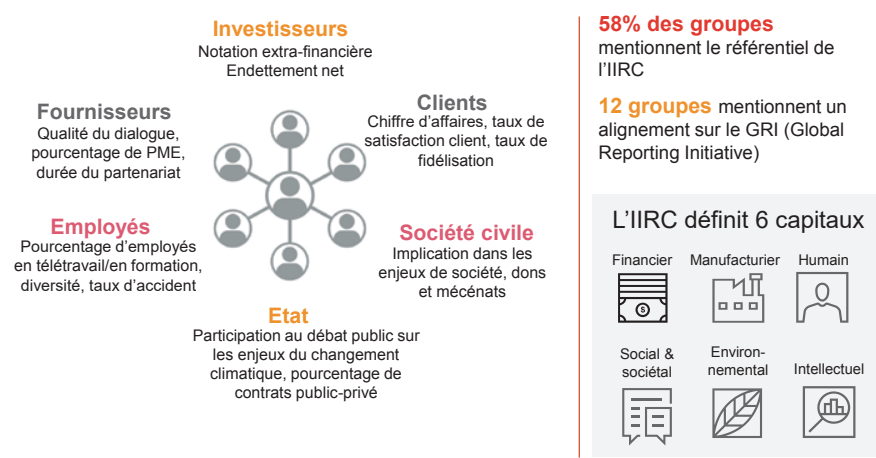

Source: PwC, Rapport intégré: les pratiques des sociétés cotées en 2019 (p. 6).

On rejoint ainsi la pensée de Peter F. Drucker, théoricien du management autrichien puis naturalisé américain, pour qui la finalité de l'entreprise se définit aussi au regard des services rendus à la clientèle. Dans tous les cas, la maximisation des bénéfices ne peut donc être la seule raison d'être de l'entreprise, ce qui explique l'importance croissante accordée à la RSE.

L'information réglementée, initialement limitée à des déclarations de nature purement financière, s'est donc 
élargie au fil du temps à des considérations nouvelles, "extrafinancières», à mesure de l'évolution des cadres normatifs à l'échelle mondiale, européenne et nationale.

\section{Quelles conséquences pour les linguistes amenés à traduire I'information extra-financière?}

\section{De la rédaction à la traduction \\ de l'information extra-financière}

Les traducteurs spécialisés en communication d'entreprise ont donc vu émerger ces dernières années de nouveaux types de publications à traduire et un nouveau vocabulaire, à mesure de l'élargissement du périmètre d'informations à fournir de manière plus ou moins obligatoire. En 2013 déjà, la revue Traduire consacrait un numéro à l'environnement comme spécialisation durable ${ }^{8}$. Sept ans plus tard, une partie des constats alors établis demeurent valables, même si certains usages ont commencé à se généraliser et ne font (presque) plus débat. En effet, le DD, la RSE, les critères ESG ${ }^{9}$ sont autant de sigles qui sont venus s'ajouter au traditionnel jargon financier des rapports d'entreprise.

Comme souvent lors de l'apparition d'un nouveau domaine de spécialisation, les linguistes font face à des problèmes d'ordre terminologique et phraséologique. Les considérations environnementales, en particulier, font appel à un vaste champ de connaissances scientifiques (biologie, météorologie, chimie...) parfois émergentes, et comme souvent à notre époque, majoritairement étayées par un corpus de textes rédigés en anglais - y compris par des locuteurs non natifs.

Une autre difficulté des rapports de développement durable réside dans la multiplicité des rédacteurs, et parfois, des traducteurs. En effet, ces publications volumineuses (de 50 à 200 pages pour un rapport intégré, à plus de 400 pages pour un document de référence) sont élaborées à plusieurs

8. journals.openedition.org/traduire/196, consulté le 29/05/2020.

9. Environnement, société, gouvernance. 
mains, dans des délais souvent très courts, une situation qui présente avantages et inconvénients.

D'un côté, la récurrence de ce type de publication et la politique de bilinguisme adoptée par la plupart des grandes entreprises offrent généralement aux linguistes qui travaillent sur un rapport de développement durable pour un nouveau client un corpus de référence tout trouvé. En effet, de larges passages sont repris et/ou adaptés d'une année sur l'autre, et certains paragraphes «nouveaux» se retrouvent dans les autres publications du groupe (communiqués de presse, par exemple).

De l'autre, la distinction entre version cible et version source a tendance à s'estomper. Il est rare que le document de référence d'un grand groupe soit intégralement rédigé dans une langue, puis traduit. II est plus fréquent que les différents chapitres soient rédigés en français ou en anglais selon les directions/rédacteurs sollicités, et traduits avant même d'être stabilisés dans leur version «originale».

Dans ce contexte et en nous appuyant sur des exemples glanés au cours de nos travaux dans le domaine du développement durable, il nous a semblé intéressant de mettre en lumière le phénomène de néologisme, qui passe par l'appropriation d'un concept et le calque, mais aussi les cas plus complexes de transposition où le choix des termes a une portée culturelle ou idéologique.

\section{L'appropriation d'un concept}

Derrière les termes se cachent des concepts, c'est pourquoi les terminologues insistent autant sur la précision des définitions que sur celles des équivalents de traduction. Dans cette optique, la Commission d'enrichissement de la langue française s'est employée, dès les années 2000 , à formaliser en français la définition d'un certain nombre de termes liés au développement durable, rassemblés, à l'occasion de la COP21, dans un fascicule intitulé Vocabulaire du développement durable.

On y constate la prédominance de termes issus de domaines de spécialité scientifiques ou techniques (hydraulique et hydrologie, véhicules polluants, énergies fossiles et bioénergie, sécurité nucléaire, etc.). 
Sans que cela ne nuise en rien à la pertinence de l'analyse de Jean-François Allain ${ }^{10}$, on y apprend que le terme développement durable est acté depuis 2009 comme équivalent de sustainable development. Et pour rassurer celles et ceux qui craignent de tomber dans le piège du calque (voir ciaprès), la durabilité est acceptée depuis 2006 comme équivalent de sustainability!

Plus récemment, et après de longs débats sur la Toile", la Commission a tranché sur l'équivalent des termes decarbonation et decarbonisation/decarbonization ("ensemble des mesures et des techniques permettant de réduire les émissions de dioxyde de carbone»), alors que cohabitaient dans les publications françaises des entreprises les concurrents décarbonation et décarbonisation: ce dernier terme commençait d'ailleurs à se démarquer dans l'usage, probablement sous l'influence du mot anglais, avec toutefois un inconvénient de taille, celui d'évoquer l'inversion du processus de carbonisation ${ }^{12}$. Qu'on se le dise, depuis le 24 septembre 2019, le terme français officiel est décarbonation ${ }^{13}$. L'usage suivra-t-il?

\section{Le calque}

Toutefois, on ne peut nier que l'enrichissement de la langue française par de nouveaux termes est un processus long (pour ne pas dire lent). Dans l'intervalle, le développement durable et la RSE, comme de nombreuses autres thématiques, n'échappent pas aux calques. Le corpus scientifique et normatif étant majoritairement rédigé en anglais, il n'est pas rare de voir émerger en français des expressions qui «sentent la traduction».

Par exemple, nombre d'entreprises évoquent aujourd'hui le risque d'extreme climate events, le changement climatique figurant en bonne place parmi les préoccupations de la société, mais aussi, de plus en plus, de la sphère économique.

10. journals.openedition.org/traduire/576, consulté le 29/05/2020.

11. Voir WWW.question-orthographe.fr/question/decarbonation-ou-decarbonisation, mais aussi wWw.academie-francaise.fr/j-eudes-m-france, sites consultés le 29/05/2020.

12. fr.wikipedia.org/wiki/Décarbonation, consulté le 29/05/2020.

13. JORF $n^{\circ} 0222$ du 24 septembre 2019. 
II n'est pas rare de voir ce mot traduit par événements climatiques extrêmes, alors que l'on pourrait avantageusement employer les termes épisodes, phénomènes ou encore manifestations climatiques (à ne pas confondre avec les «manifestations pour le climat»!).

Plus surprenant, le terme ecologist, récemment repéré dans un rapport de développement durable de l'un de nos clients et inopportunément traduit par écologiste, alors qu'il s'agissait d'un écologue! Or, le premier milite pour la protection de l'environnement, tandis que le second analyse l'impact des activités humaines sur l'environnement et la biodiversité. On a l'intuition ${ }^{14}$ que le suffixe employé change le sens et la connotation du terme en français - l'écologiste étant le sectateur d'une opinion et l'écologue un spécialiste scientifique.

C'est aux linguistes vigilants qu'il reviendra au cas par cas de trancher entre un calque dont l'emploi est entré dans l'usage et une véritable erreur de sens qu'il conviendra de corriger.

\section{Le choix des termes: un acte culturel et idéologique}

Si l'usage de certains termes, qu'ils fassent l'objet d'une appropriation ou d'un calque, est désormais figé, d'autres continuent de susciter le débat. Là encore, le choix d'un terme n'est pas une simple manie de linguiste à la recherche du «mot juste», mais un acte fort, souvent chargé sur le plan idéologique.

Les différentes sensibilités culturelles rendent difficile la traduction de certains concepts-clés de la RSE. Car, comme le dit François Vezin, traducteur de Heidegger: "Y a-t-il [...] une véritable distinction à faire entre traduction et traduction philosophique ${ }^{15}$ ? 》

Prenons l'exemple du terme anglais human rights: l'ancrage historique de notre inestimable "Déclaration des droits de l'Homme et du citoyen » de 1789 nous porterait à le traduire par droits de l'Homme, avec une majuscule pour signifier

14. Intuition qui peut être parfois démentie: www.researchgate.net/publication/278623900_ Composition_neoclassique_en_-logue_et_en_logiste_les_noms_en_-logue_sont_ils_ encore_des_noms_de_specialistes, consulté le 29/05/2020.

15. www.cairn.info/revue-philosophique-2005-4-page-489.htm, consulté le 29/05/2020. 
que l'on fait référence à l'ensemble de l'humanité. Pourtant, le recours au mot homme (ou Homme, dont la majuscule ne s'entend pas à l'oral) est de plus en plus contesté par celles et ceux qui défendent les droits des femmes et qui dénoncent un manque de représentativité de la langue. L'écriture inclusive, née d'une volonté de faire changer les mentalités sur les représentations des hommes et des femmes, privilégie l'expression droits humains qui, si elle sonne encore à certaines oreilles comme un vilain anglicisme, tend à se généraliser dans la plupart des grandes institutions et $O N G$ françaises (par exemple Humanité \& Inclusion - anciennement Handicap International).

La dimension culturelle jove aussi un rôle central dans la perception de certains concepts relevant du champ de la RSE, tels que philanthropy - «du grec philanthrôpia; sentiment qui pousse les hommes à venir en aide aux autres; amour de l'humanité (la lecture du Larousse est parfois si simple et si belle...). Pour savoir s'il doit parler de philanthropie, de mécénat d'entreprise ou encore d'entreprise citoyenne ou écoresponsable, le linguiste doit faire preuve d'une compréhension fine des enjeux sociopolitiques recouverts par les termes dans leur contexte: une définition plus actuelle du terme philanthropie nous fait prendre quelques distances avec la pureté étymologique pour parler de dons d'argent, de ressources matérielles et non matérielles, accordés par des individus ou des entreprises afin d'affronter des problématiques sociétales. Cette définition est parfaitement ancrée dans la langue anglaise, et particulièrement dans la culture nord-américaine, adepte de la corporate philanthropy.

En France, on aurait plutôt tendance à parler de mécénat d'entreprise. La Fondation de France, qui incarne la vitalité du mouvement des fondations "à l'américaine» dans notre pays, définit ainsi le mécénat: "Soutien matériel apporté sans contrepartie directe de la part du bénéficiaire, à une œuvre ou à une personne pour l'exercice d'activités présentant un intérêt général.»

"Sans contrepartie directe» donc, même si les contreparties indirectes attendues sont celles de la valorisation de la réputation, mais aussi de la réduction d'impôts. En France, pour inciter les entreprises au don, la loi Aillagon sur le mécénat leur permet, depuis 2003 , de défiscaliser $60 \%$ 
des dons, dans la limite de 0,5\% du chiffre d'affaires. Pour les particuliers, ce seuil est porté à $66 \%$, dans la limite de $20 \%$ du revenu imposable. Aux États-Unis, l'article $501 c 3^{16}$ du Code des impôts (Internal Revenue Code) permet $100 \%$ d'exonération des dons monétaires. Les dons de Bill Gates ou Mark Zuckerberg à des fondations gérées par eux-mêmes nous éloignent alors sensiblement de la définition donnée par le Larousse. La philanthropie est donc une démarche volontaire, le plus souvent liée au bon vouloir des dirigeants des entreprises et à leurs préoccupations personnelles.

Cette suspicion sur la sincérité de la générosité des donateurs, en particulier des entreprises, a suscité de vifs débats au lendemain de l'incendie de Notre-Dame de Paris le 15 avril 2019. La journaliste Nicole Vulser résume la question du mécénat en ces termes: "Donner c'est à la fois prouver sa richesse, sa générosité, sa solidarité et sa puissance tout en améliorant son image et en faisant le bien», et reprend les propos de Bernard Arnault, qui avait annoncé un don de 200 millions d'euros sans prétendre à la défiscalisation: " "C'est assez consternant de voir qu'en France on se fait critiquer même quand on fait quelque chose" qui est "une preuve d'intérêt général" [...] "Dans certains pays, on serait plutôt félicités." »

Élisa Chelles, qui a étudié les dynamiques sociopolitiques de la philanthropie aux États-Unis et en France, écrit quant à elle:

La philanthropie serait ici [aux États-Unis] une forme valorisée de salut gagé sur un engagement civique [...], et là [en France] l'envers d'un monopole d'État sur les formes de solidarité nationale, un partage conforté par le travail de socialisation politique et les cadres ordinaires du civisme.

Au-delà des termes employés, il ne faut donc jamais oublier le contexte (linguistique et culturel), les intentions du rédacteur qui emploie tel mot plutôt que tel autre, et le public visé.

16. WWW.irs.gov/charities-non-profits/charitable-organizations/exemption-requirements501c3-organizations, consulté le 29/05/2020. 


\section{Conclusion: du réglementaire au linguistique et du linguistique au culturel}

Quoi qu'il en soit, notre rôle de linguiste consiste à nous informer de l'évolution de l'usage des termes car, après tout, nous sommes spécialistes des langues vivantes! Dans la mesure du possible, nous pouvons conseiller nos clients en les orientant vers les termes consacrés dans le domaine du développement durable et de la RSE, même si nous devrons toujours tenir compte de leurs préférences internes.

De plus, comme nous avons tâché de le montrer dans cet article, la RSE est une approche holistique de l'entreprise. Véritable phénomène de société, elle touche une telle diversité de sujets que tous les traducteurs et traductrices, quel que soit leur domaine de spécialisation, y seront vraisemblablement tôt ou tard confrontés.

chloe@delhom-traduction.com contact@audreyprost-traduction.com

Diplômée de I'ISIT en 2011, Chloé Delhom a suivi un deuxième cursus en management RSE et travaillé cinq ans dans ce domaine en entreprise, puis en tant que consultante RSE dans un cabinet de conseil. En 2017, elle a choisi d'exercer en tant que traductrice et interprète indépendante, de l'anglais et de l'espagnol au français, avec le développement durable comme domaine de prédilection.

Diplômée de l'ISIT en 2011, Audrey Prost a travaillé cinq années en entreprise et en institution sur des sujets économiques et financiers, avant de devenir traductrice indépendante, de l'anglais et de l'allemand au français, en 2015. Aujourd'hui, elle traduit et révise des documents de communication institutionnelle pour des clients variés. Membre d'AlumnISIT et de la SFT, Audrey est aussi secrétaire d'Aprotrad. 
Autorité des marchés financiers (AMF), Guide relatif au dépôt de linformation réglementée auprès de l'AMF et à sa diffusion, France, 2013, www.amf-france.org/sites/ default/files/contenu_simple/guide/guide_professionnel/Guide $\% 20$ relatif $\% 20$ au $\% 2$ odepot $\% 20$ de $\% 201 \% 27$ information $\%$ 20reglementee $\% 20$ aupres $\% 20$ de $\% 20$ $1 \% 27$ AMF\%20et\%20a\%20sa\%2odiffusion.pdf, consulté le 2/06/2020.

BEINEIX Olivier, DESMIER Bertrand, «Du reporting RSE à la déclaration de performance extra-financière. Décryptage», France, RSE Reporting, 4 août 2017, rse-reporting.com/reporting-rse-declaration-performance-extra-financiere, consulté le 2/06/2020.

CHELLE Élisa, «La philanthropie aux États-Unis et en France, retour sur une traditionnelle opposition », in Sociologie, France, vol. 8, n 4, 2017, p. 395-408, www. cairn.info/revue-sociologie-2017-4-page-395.htm, consulté le 2/06/2020.

Comité international de l'information intégrée (IIRC), Cadre de référence international portant sur le reporting intégré, Royaume-Uni, 2013, integratedreporting.org/wp-content/uploads/2015/03/13-12-08-THE-INTERNATIONAL-IRFRAMEWORKFrench.pdf, consulté le 2/06/2020.

Commission d'enrichissement de la langue française, Vocabulaire du développement durable, France, 2015, www.culture.fr/eng/Ressources/FranceTerme/ Actualites/Le-vocabulaire-du-developpement-durable-novembre-2015, consulté le $2 / 06 / 2020$.

DE GIOVANNI Stéphanie, LÊ HOROVITZ Olivia, SIMON Nicolas, «Évolution législative de fond: la montée en puissance des critères extra-financiers », France, Simon Associés, 2019, www.simonassocies.com/evolution-legislative-de-fond-lamontee-en-puissance-des-criteres-extra-financiers, consulté le 2/06/2020.

Fondation de France, L'entreprise engagée: nouvelles frontières de la RSE et du mécénat, France, 2018, www.fondationdefrance.org/sites/default/files/atoms/files/1_entreprise_engagee_nouvelles_frontieres_du_mecenat_et_de_la_rse.pdf, consulté le 2/06/2020.

FOURNIER Catherine, «Non, Mark Zuckerberg ne donne pas toute sa fortune à des œuvres caritatives », in France Info, France, 2015, www.francetvinfo.fr/internet/reseaux-sociaux/facebook/non-marc-zuckerberg-ne-donne-pas-toute-safortune-a-sa-fondation-caritative_1201855.html, consulté le 2/06/2020.

Haut Conseil à l'Égalité entre les hommes et les femmes, "Droits humains" vs "Droits de l'Homme" : en finir avec une logique linguistique discriminatoire", France, 10 décembre 2018, haut-conseil-egalite.gouv.fr/parite/actualites/article/ droits-humains-une-expression-qu-il-est-temps-de-generaliser, consulté le 2/06/2020.

Institut français des administrateurs (IFA), Le conseil d'administration et le reporting intégré, France, 2017, www.ifa-asso.com/fileadmin/user_upload/GT_A5_ ReportingIntegre2017_vdef@.pdf, consulté le 2/06/2020.

PwC, Cadre et pratiques de communication financière, France, 2019, www.pwc.fr/fr/ assets/files/pdf/2019/07/fr-france-guide-cadre-et-pratiques-de-communicationfinanciere-2019.pdf, consulté le 2/06/2020. 
PwC, Rapport intégré: les pratiques des sociétés cotées en 2019, France, 2019, www.pwc. $\mathrm{fr} / \mathrm{fr} /$ publications/communication-financiere/rapport-integre-les-pratiques-dessocietes-cotees-en-2019.html, consulté le 2/06/2020.

RIBOUD Franck, «La crise impose de repenser le rôle de l'entreprise», in Le Monde, France, 2 mars 2009, www.lemonde.fr/idees/article/2009/03/02/la-criseimpose-de-repenser-le-role-de-l-entreprise-par-franck-riboud_1162147_3232. html, consulté le 2/06/2020.

VULSER Nicole, «Notre-Dame de Paris: l'élan de générosité décrié des grandes entreprises", in Le Monde, France, 19 avril 2019, www.lemonde.fr/ idees/article/2019/04/19/notre-dame-suspicions-autour-d-un-elan-de-generosite_5452319_3232.html, consulté le 2/06/2020. 